\title{
Efficient balancing by effort-based activation of demand response services
}

\author{
Felix N. Claessen*†, Michael Kaisers* and Han La Poutré*‡ \\ * Centrum Wiskunde \& Informatica (CWI) \\ $\dagger$ Seita B.V. \\ $\ddagger$ Technical University Delft (TU Delft) \\ Amsterdam, The Netherlands \\ Amsterdam, The Netherlands \\ Delft, The Netherlands
}

\begin{abstract}
We present a unified model for flexibility services in the power system, identify two existing categories (ramping and loading) and introduce a new category (stalling). Each service is characterised by duration, capacity and effort, with associated prices. We show that the effort of stalling, measurable in $\mathrm{kWh}^{2}$, is a significant cost component for balancing through storage and demand response (DR). In future energy systemswith increased reliance on renewable generation-storage and DR resources are expected to become an important component of power system flexibility and balancing. Existing resource allocation mechanisms are mainly based on pricing in $\mathrm{kW}$ (for ramping) and in $\mathrm{kWh}$ (for load). Simulations demonstrate that conventional pricing mechanisms yield inefficient allocations of storage and DR resources for power balancing. In contrast, we show that introducing an additional pricing component in $€ / \mathbf{k W h}{ }^{2}$ (for stalling) improves the allocation efficiency.
\end{abstract}

\section{INTRODUCTION}

The power system requires a continuous balance of demand and supply. Traditionally, the natural variation in energy demand is resolved by ramping the power output of large generators up and down. Societies now wish to replace conventional generators with renewable resources. Most of these resources do not have ramping capabilities, and will in fact contribute to the variation of load in the system. An important component of flexibility in future power systems must come from storage and demand response (DR). Resources that offer that kind of flexibility are able to shift load over time, but usually not willing to change their net consumption over a longer period of time. We are interested in mechanisms that can activate this largely untapped flexibility in an efficient way.

Current mechanisms settle the use of power system flexibility by the amount of energy (in $\mathrm{kWh}$ ) that a resource has withheld (or delivered extra) by reducing (or increasing) its load. This is a useful quantity for conventional generators, whose variable costs for providing flexibility also scale with the generated amount of $\mathrm{kWh}$. That is, the effort that a conventional power plant has to spend to resolve an imbalance is proportional to its variable production costs in $€ / \mathrm{kWh}$.

In contrast to the cost of producing energy, the cost of storing energy or delaying consumption depends on the amount of energy and the amount of time. Therefore, applying the same pricing structure to reward power plants, storage operators and consumers for their flexibility (i.e. by settling in $\mathrm{kWh}$ ) yields an unfair bias, because the pricing structure is more congruent with the cost structure of one technology.

Outlook: In this paper we present a unified model for electricity services, investigate the pricing structure of various services in relation to their cost structure, and show how storage and DR services can be activated more efficiently by including duration in their price. In Section II we elaborate this paper's contributions and its relation to the literature. In Section III we formalise our model and introduce definitions for shifting energy over time. In Section IV we investigate an alternative pricing structure for balancing that captures the effort of storage and DR. Section V concludes the article.

\section{PRELIMINARIES}

In this paper we look at the power system as a set of energy technologies that provide electricity services to each other. We regard services as "activities that are the object of exchange" [1]. Electricity services comprise the entire chain of activities that balance power demand and supply over space and time. Our taxonomy is given in Figure 1.

Separate legal treatment of the commodity of electricity (as a marketable good) and its balancing and transport (as ancillary and system services) has been designated by European courts, to apply the concept of free movement of goods (within the EU's internal market) to electricity production and retail [2]. In addition, typology, terminology and formal definitions of ancillary services vary considerably according to regional custom [3]. For our concern, it is not important to make these distinctions (e.g. between goods and services), because we can capture all activities that balance power demand and supply in one service model.

Service parameters of ancillary services are mostly geared towards generator limitations rather than load limitations [3, p.33]. For example, maximum service duration is not a service parameter, whilst an essential component of load flexibility. As a result, DR resources have a hard time providing conventional ancillary services. In order to successfully transition from a power system with supply flexibility (from conventional resources) to a system with demand and storage flexibility (and renewable resources), new service definitions that include parameters for demand-side resource constraints are markedly needed.

This paper's first contribution is a unified model characterising all activities in Figure 1 as a service. ${ }^{1}$ We restructure the historical taxonomy and refocus on individual servicesprovided by flexible resources-that support the essential

\footnotetext{
${ }^{1}$ We do not distinguish between the concepts of electricity service, flexibility service and balancing service, since each of these services entails a modulation of power flow over space and time.
} 


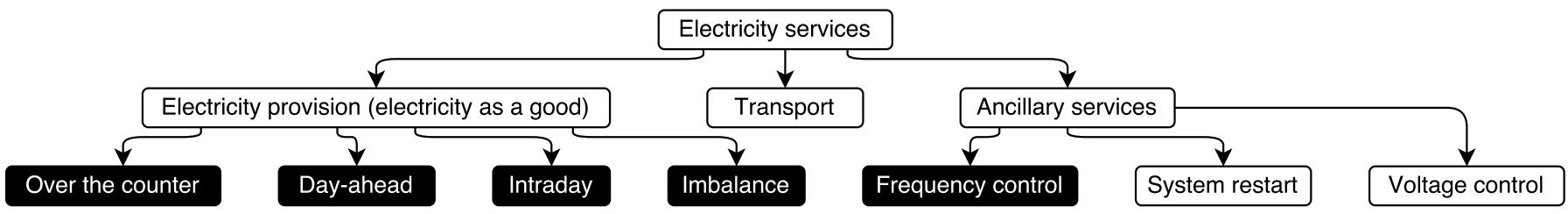

Fig. 1: One of many possible categorisations of electricity commodities, from the perspective of our proposed unified model of services. The new stall service category is most pertinent to the blackened areas for which storage and DR may play a significant role.

function of the power system: a continuous balance of power demand and supply over space and time. We identify two categories of individual services, with existing applications as a marketable commodity:

1) Load services provide a desired amount of energy (in $\mathrm{kWh}$ ) within a desired time interval.

E.g. electricity provision and transport.

2) Ramp services provide a desired increase (or decrease) of power (in $\mathrm{kW}$ ) within a desired time interval.

E.g. frequency control and system restart services.

We then introduce a third category, with potential applications as a marketable commodity:

3) Stall services provide a desired shift of energy - for which we propose a new metric (in $\mathrm{kWh}^{2}$ )—within a desired time interval.

E.g. storage and demand response.

Our model allows each of these services to be captured by a generic set of parameter definitions when applied on timederivatives of energy profiles.

The metric we propose to quantify stall services is the first order integral of energy over time, with units in $\mathrm{kWh}^{2}$ (or Js, i.e. Joule-second). This concept stems from a combination of queueing theory and what we frame as integral dynamics. Integral dynamics is the nascent study of time-integrals of state parameters in classical mechanics, which originated from the concept of absement, the first order time-integral of displacement. $^{2}$ Time-integrals of energy have previously been used as a metric for human reflex performance [4] and for memory properties in electrical components [5]. The directly related concept in queueing theory is that of traffic volume, which can be expressed in call-seconds, kilometer-minutes, erlang-hours, etc. [6, p.106]. Essentially, we measure the traffic volume of energy, e.g. through a battery.

Our second contribution is to show that our new metric for stall services captures a significant variable cost component of balancing through storage and DR. Resources that can provide storage or DR capabilities are limited by their response duration and "tend to have [variable] energy cost curves that rise dramatically with storage duration" [3, p.34]. Our suggestion is to include duration as a component of service costs.

\footnotetext{
${ }^{2}$ This largely unexplored field of study was initially coined as integral kinematics due to a focus on integrals of distance. We suggest integral dynamics to capture the study of time-integrals of displacement (integral kinematics), momentum (integral statics) and energy (integral kinetics).
}

Recent attempts to define new flexibility services that are more suitable for storage and DR indeed tend to include specific parameters for service duration, but do not use duration as a scalar of variable costs explicitly [7], [8], [9].

Finally, our third contribution is to show that balancing becomes more efficient when the pricing structure of storage and DR services explicitly includes the usage duration of the stall service in its variable costs. To investigate this hypothesis, we simulate a DR mechanism (between a retailer and a customer) that uses either a conventional tariff with variable costs for load services (in $€ / \mathrm{kWh}$ ), or our innovative tariff with additional variable costs for stall services (in $€ / \mathrm{kWh}^{2}$ ).

\section{A SERVICEABLE MODEL OF POWER BALANCING}

Our service model consists of two components. In Section III-A we introduce a general model to price services. In Section III-B we apply our model to the power system and define six balancing services.

\section{A. Service terminology: duration, capacity and effort}

We calculate total service $\operatorname{costs} c_{\mathrm{s}}$ as the sum of fixed costs $c_{T}$, investment costs $c_{C}$ and variable costs $c_{S}$, according to:

$$
\begin{aligned}
c_{\mathrm{s}} & =c_{T}+c_{C}+c_{S} \\
\text { where } \quad c_{T} & =\mathbf{p}_{T} \cdot T, \quad c_{C}=\mathbf{p}_{C} \cdot C, \quad c_{S}=\mathbf{p}_{S} \cdot S
\end{aligned}
$$

The parameter $T$ is the duration for which the service may be used, $C$ is the capacity denoting the maximum possible service rate, and $S$ is the effort denoting the total use of service, as illustrated in Figure 2. Associated unit prices are: the service availability price $\mathbf{p}_{T}$, the service capacity price $\mathbf{p}_{C}$, and the service effort price $\mathbf{p}_{S}$, respectively.

Let $s(t) \in[0, C]$ be the rate at which a service is performed at time $t \in[0, T]$. We define the service effort $S$ as:

$$
S=\int_{0}^{T} s(t) \mathrm{d} t
$$

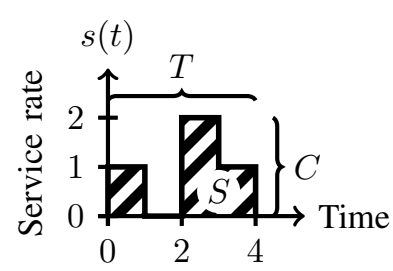

$$
\begin{aligned}
T & =4 & & \text { Duration } \\
C & =2 & & \text { Capacity } \\
S & =4 & & \text { Effort } \\
C_{F} & =1 / 2 & & \text { Capacity factor } \\
\bar{W} & =4 / 3 & & \text { Mean service time } \\
\bar{L} & =1 & & \text { Mean queue length } \\
\bar{\lambda} & =3 / 4 & & \text { Mean arrival rate }
\end{aligned}
$$

Fig. 2: Example parameters for a time profile of service usage. 
The capacity factor $C_{F} \in[0,1]$ indicates the utilisation of potential output. For a service $s(t)$ used up to time $T$, potential output is $C \cdot T$, and realised output is the effort $S$, such that:

$$
C_{F}=\frac{S}{C \cdot T}
$$

Furthermore, the total service time $W$ (full-capacity operating hours) and average service time $\bar{W}$ are given by:

$$
\text { where } \quad \begin{aligned}
W & =C_{F} \cdot T \quad, \quad \bar{W}=\frac{\bar{L}}{\bar{\lambda}} \\
\text { wh } & =\frac{S}{T}=\frac{1}{T} \cdot \int_{0}^{T} s(t) \mathrm{d} t \\
\text { and } \quad \bar{\lambda} & =\frac{1}{T} \cdot \int_{0}^{T} \dot{s}(t) \cdot[\dot{s}(t)>0] \mathrm{d} t
\end{aligned}
$$

where $\bar{L}$ is the average service rate (mean queue length), $\bar{\lambda}$ is the average increase in service rate (mean arrival rate), and $\dot{s}(t)$ is the change in service rate at time $t .{ }^{3} \mathrm{Eq} .6$ is analogous to Little's law for queueing processes.

\section{B. Balancing services: ramping, loading and stalling}

The application of our service model to power balancing produces the variables and parameters in Table I. We first explain the relationship between the variables, then define our service parameters and end with examples.

Energy variables: Let $x(t)$ be the total amount of electrical work (in $\mathrm{kWh}$ ) that an agent has done on the grid up to time $t \in[0, T]$. A positive $x(t)$ denotes net production until time $t$, and negative $x(t)$ denotes net consumption. Following Newton's notation for time-derivatives, $\dot{x}(t)$ represents the power flow (in $\mathrm{kW}$ ) at time $t$, where positive $\dot{x}(t)$ denotes a production rate and negative $\dot{x}(t)$ denotes a consumption rate. Similarly, $\ddot{x}(t)$ represents the acceleration of power flow (in $\mathrm{kW} / \mathrm{h}$ ) at time $t$, where positive $\ddot{x}(t)$ denotes an increasing production rate (or decreasing consumption rate) and negative $\ddot{x}(t)$ denotes a decreasing production rate (or increasing consumption rate). Finally, we define the agent's absergy ${ }^{4}$ in $\mathrm{kWh}^{2}$ ) at time $t$ as:

$$
X(t)=\int_{0}^{t} x(\tau) \mathrm{d} \tau+X_{0}
$$

where $X_{0}$ is the initial absergy at $t=0$. Absergy is a useful metric for storage and DR. It measures the agent's traffic volume [6, p.106] in terms of energy volume (of production or consumption) and the duration in which a given volume is kept. Positive $X(t)$ denotes that most of the agent's traffic up to time $t$ was due to lending energy to the grid, while negative $X(t)$ denotes that most traffic was due to the agent borrowing energy from the grid. Both advancing production and delaying consumption increases the agent's absergy. Both

\footnotetext{
${ }^{3}$ Iverson bracket notation is used in equations throughout this article (the bracketed term is 1 if the condition inside is satisfied, and 0 otherwise).

${ }^{4}$ In analogy with the concept of absement [4], a portmanteau of "absence of displacement" which indicates the time-integral of position (hence in units of ms or meter-seconds), we use the term absergy as a portmanteau of "absence of energy" to indicate the time-integral of energy (in SI units of Js, or in $\mathrm{kWh}^{2}$ to maintain our orientation on power networks).
}

TABLE I: Units and corresponding variables and parameters in our service model for power balancing.

\begin{tabular}{lccc}
\hline Unit & Variable & $C$ & $S$ \\
\hline $\mathrm{kWh}^{2}$ & $X$ & Action capacity & Stall effort $S_{x}$ \\
$\mathrm{kWh}$ & $x$ & Stall capacity $C_{x}$ & Load effort $S_{\dot{x}}$ \\
$\mathrm{~kW}$ & $\dot{x}$ & Load capacity $C_{\dot{x}}$ & Ramp effort $S_{\ddot{x}}$ \\
$\mathrm{~kW} / \mathrm{h}$ & $\ddot{x}$ & Ramp capacity $C_{\ddot{x}}$ & Surge effort \\
\hline
\end{tabular}

delaying production and advancing consumption (and thus, storing) decreases the agent's absergy.

Service parameters: Given the energy variables in Table I, we define two services for each of three categories (ramping, loading and stalling). For ramping, we define an upramp service at rates $s_{\ddot{x}}^{\uparrow}$ and a downramp service at rates $s_{\ddot{x}}^{\downarrow}$, according to:

$$
s_{\ddot{x}}^{\uparrow}(t)=\ddot{x}(t) \cdot[\ddot{x}(t)>0] \quad, \quad s_{\ddot{x}}^{\downarrow}(t)=\ddot{x}(t) \cdot[\ddot{x}(t)<0]
$$

and use Eq. 3 to determine the effort $S_{\ddot{x}}$ for both ramp services. The required capacity $C_{\ddot{x}}$ is at least $\max s_{\ddot{x}}(t) \forall 0 \leq t \leq T$. Other parameters in Table I are determined in a similar fashion, using the following definitions for upload and download services, and upstall and downstall services, at rates $s_{\dot{x}}^{\uparrow}, s_{\dot{x}}^{\downarrow}$, $s_{x}^{\uparrow}$ and $s_{x}^{\downarrow}$, respectively:

$$
\begin{array}{rlrl}
s_{\dot{x}}^{\uparrow}(t) & =\dot{x}(t) \cdot[\dot{x}(t)>0] & , & s_{\dot{x}}^{\downarrow}(t)=\dot{x}(t) \cdot[\dot{x}(t)<0] \\
s_{x}^{\uparrow}(t)=x(t) \cdot[x(t)>0] & , & s_{x}^{\downarrow}(t)=x(t) \cdot[x(t)<0]
\end{array}
$$

In Eq. 11, the arrows indicate whether the agent is providing an upload service (production) or using a download service (consumption). Finally, in Eq. 12, the arrows indicate whether the agent is providing an upstall service $s_{x}^{\uparrow}(t)$ (e.g. advancing production) or a downstall service $s_{x}^{\downarrow}(t)$ (e.g. storage). ${ }^{5}$

Example 1-Load and ramp services: Consider a small factory that uses a $100 \mathrm{~kW}$ machine once a day for 2 hours, and balances its load through a monthly supply service from service provider A. In a month, A will provide an upload service with:

- a duration $T_{\dot{x}}^{\uparrow}$ of 30 days, and service time $W_{\dot{x}}^{\uparrow}$ of $60 \mathrm{~h}$,

- an upload capacity $C_{\dot{x}}^{\uparrow}$ of $100 \mathrm{~kW}$, and

- an upload effort $S_{\dot{x}}^{\uparrow}$ of $\left(30\right.$ days $\left.\cdot 200 \frac{\mathrm{kWh}}{\text { day }}\right)=6 \mathrm{MWh}$.

The variation of the load requires upramping when the machine is turned on, and downramping when it is turned off again. If both ramps would take 8 minutes, the supply service of A would also entail a ramp service with:

- service times $W_{\ddot{x}}^{\uparrow}$ and $W_{\ddot{x}}^{\downarrow}$ of $4 \mathrm{~h}$,

- ramp capacities $C_{\ddot{x}}^{\uparrow}$ and $C_{\ddot{x}}^{\downarrow}$ of $\left(\frac{100 \mathrm{~kW}}{8 \mathrm{~min}}\right)=750 \mathrm{~kW} / \mathrm{h}$, and

- ramp efforts $S_{\ddot{x}}^{\uparrow}$ and $S_{\ddot{x}}^{\downarrow}$ of $\left(30\right.$ days $\left.\cdot 100 \frac{\mathrm{kW}}{\text { day }}\right)=3 \mathrm{MW}$.

${ }^{5}$ Table I includes two additional parameters for completeness, and their names should be considered suggestions without much claim: Action capacity indicates the maximum amount of possible stall within a given time horizon. For example, a flexible prosumer may be willing to delay up to $10 \mathrm{kWh}$ for up to 2 hours within a given day, which would yield an action capacity of 20 $\mathrm{kWh}^{2}$. The second quantity, surge effort, indicates the effect on ramp rate (in $\mathrm{kW} / \mathrm{h}$ ) of a jerk in power flow. However, we won't use these concepts further. 


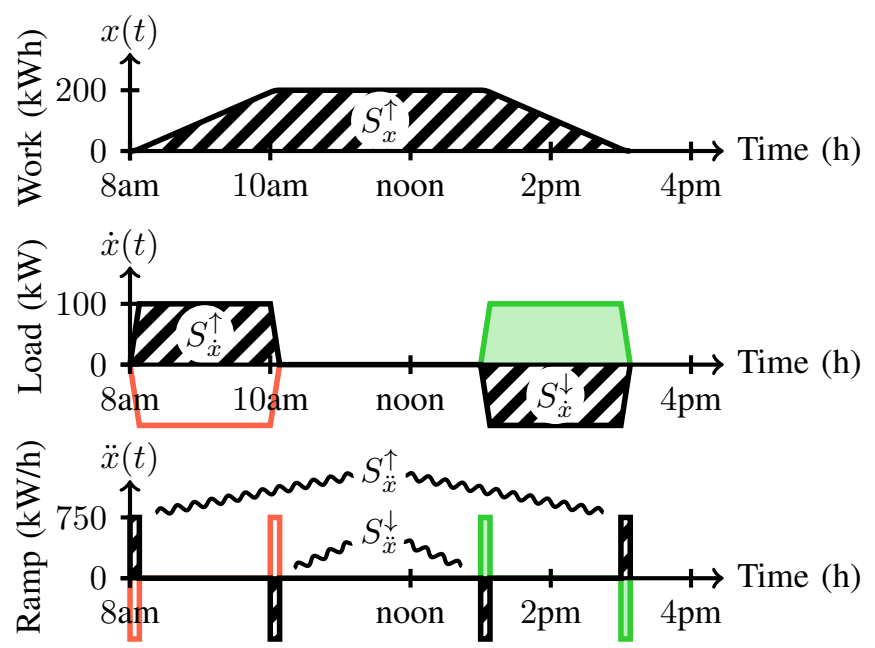

Fig. 3: Three derivative perspectives of a DR service (hatched) distinguishing a ramp service (bottom), a load service (middle) and a stall service (top), jointly provided by service provider B. By lending energy for 5 hours, this DR service would resolve the misaligned supply from service provider A (shaded green) with the factory's demand (red) in our example.

However, it is very uncommon to charge fees to small end users for ramp rates, or to even inform them on the matter.

Example 2-Stall services: Now let us extend our example by assuming that there is a consistent mismatch in the timing of the factory's demand and the timing of supply. For example, service provider A can only deliver between $1 \mathrm{pm}$ and $3 \mathrm{pm}$, while the factory requires power between $8 \mathrm{am}$ and $10 \mathrm{am}$. To resolve this, the factory might need a second service contract through which it is able to advance energy supply by 5 hours each day, e.g. a DR service from service provider $B$ that enables the factory to borrow energy from a fleet of electric vehicles (EVs). Each day, B would provide an upstall service as depicted in Figure 3, with:

- a duration $T_{x}^{\uparrow}$ of 1 day, and service time $W_{x}^{\uparrow}$ of $5 \mathrm{~h}$,

- an upstall capacity $C_{x}^{\uparrow}$ of $200 \mathrm{kWh}$, and

- an upstall effort $S_{x}^{\uparrow}$ of $(5$ hours $\cdot 200 \mathrm{kWh})=1 \mathrm{MWh}^{2}$.

Besides sufficient stall capacity, the vehicles' batteries also require sufficient load capacity, will face conversion losses and will use up a part of their cycle lifetime. The DR service of B would also entail load and ramp services with:

- load capacities $C_{\dot{x}}^{\uparrow}$ and $C_{\dot{x}}^{\downarrow}$ of $100 \mathrm{~kW}$,

- load efforts $S_{\dot{x}}^{\uparrow}$ and $S_{\dot{x}}^{\downarrow}$ of $(2$ hours $\cdot 100 \mathrm{~kW})=200$ $\mathrm{kWh}$, and

- ramp efforts $S_{\ddot{x}}^{\uparrow}$ and $S_{\ddot{x}}^{\downarrow}$ of $100 \mathrm{~kW}$.

Here, ramp effort scales with the number of charging cycles, while load effort also scales with the depth of (dis)charge, and stall effort scales with both and with the storage/shift duration. By pricing stall effort, provider B can recuperate its private costs that scale with storage/shift duration. Two real-world phenomena give rise to such costs:
Firstly, round-trip efficiency decreases with duration. The amount of this efficiency loss varies tremendously between different storage and DR resources. Although most battery technologies have negligible (intraday) dissipation losses [10], they may have a critical influence in e.g. heat storage; while for DR, shifting consumption entails a deviation from an otherwise optimised schedule. As a result, less efficient means will be selected, or more means than necessary. In the case of an EV fleet, for example, a longer DR service will necessarily involve more cars, as individual cars may drive off in between.

Secondly, opportunity costs increase with duration. The service-time spent stalling may have been used to serve some other purpose. For example, DR by industrial technologies may induce high variable costs due to delaying personnel.

\section{Demand Response as a stochastic Game}

In this section we study whether including a price component for stall effort can improve the allocation efficiency of DR services. We investigate a retailer-customer system in which a flexible customer offers to resolve imbalances on the retailer's behalf, at a known tariff $\mathcal{T}{ }^{6}$ As a pricing structure, we use various sets of unit prices $\mathbf{p}^{\mathcal{T}}$ for service duration, capacity and effort. Costs for duration and capacity correspond to fixed costs and investments, respectively, while costs for effort correspond to variable costs. Here we focus on the latter. Specifically, we show that pricing stall effort (in $€ / \mathrm{MWh}^{2}$ ), in addition to the more common pricing of load effort (in $€ / M W h$ ), increases the market efficiency of the retailer-customer system when the customer has some flexibility.

Method: We model the system as a game played by the retailer and the customer, in which exogenous events are represented as stochastic moves by a third player: nature. Similar to other authors [7], [9], [11], we let the customer set the tariff, because it has the relevant private information about costs that arise when a DR service is used. In the stage game:

- the customer offers a tariff (a set of unit prices for load and stall effort) for providing DR to the retailer,

- nature informs the retailer on upcoming imbalances w.r.t. its market commitments (where imbalances arrive one at a time, are unrelated, and are dealt with sequentially),

- for each imbalance, the retailer decides whether or not to activate the DR mechanism,

- if activated, the customer resolves the imbalance by deviating from its precommited load, and

- we keep track of the best possible allocation of resources from a social welfare perspective, as a reference.

In the repeated game, the customer:

- can change the unit prices within its set $\mathbf{p}^{\mathcal{T}}$, and

- learns which prices optimise its own utility.

Finally, we compare the resulting allocation efficiency for two different pricing structures.

\footnotetext{
${ }^{6}$ Note the perhaps subtle reversal of the roles of seller and buyer. The energy seller (i.e. the retailer) becomes a flexibility buyer, and the energy buyer (i.e. the customer) becomes a flexibility seller.
} 
Notation: Let $\mathbf{q}(X)$ be the vector of quantities that may be priced through a balancing service contract for a given imbalance $X$, where the imbalance is specified as an absergy function $X(t)$ with $t \in(0, T) .{ }^{7}$ We define a conventional tariff $\mathbf{p}_{\text {con }}^{\mathcal{T}}$ and an innovative tariff $\mathbf{p}_{\text {inn }}^{\mathcal{T}}$ that price the following quantities (load effort $S_{\dot{x}}$ and stall effort $S_{x}$ ):

$$
\mathbf{q}(X)=\left\langle S_{x}^{\uparrow}, S_{\dot{x}}^{\uparrow}, S_{x}^{\downarrow}, S_{\dot{x}}^{\downarrow}\right\rangle
$$

The conventional tariff $\mathbf{p}_{\text {con }}^{\mathcal{T}}$ always contains zero prices for stall effort. Furthermore, let $\mathbf{p}^{\mathcal{R}}$ and $\mathbf{p}^{\mathcal{C}}$ be the retailer's $\mathcal{R}$ and customer's $\mathcal{C}$ own variable costs, respectively. Then the retailer and customer have private values $u$ given by:

$$
\begin{aligned}
u^{\mathcal{C}}\left(\mathbf{p}^{\mathcal{T}}, X, a\right) & =\left(\mathbf{p}^{\mathcal{T}}-\mathbf{p}^{\mathcal{C}}\right) \cdot \mathbf{q}(X) \cdot[a=1] \\
u^{\mathcal{R}}\left(\mathbf{p}^{\mathcal{T}}, X, a\right) & =\left(\mathbf{p}^{\mathcal{R}}-\mathbf{p}^{\mathcal{T}}\right) \cdot \mathbf{q}(X) \cdot[a=1]
\end{aligned}
$$

where $a$ is the retailer's action ( $a=1$ specifies that the retailer accepts the offer, and $a \neq 1$ specifies a rejection). The retailer's policy is to accept only offers that yield a positive utility (therefore $u^{\mathcal{R}} \geq 0$ ). The customer's policy is to choose a tariff that yields the highest expected utility over the duration of the contract (therefore $\mathbb{E}\left[u^{\mathcal{C}}\right] \geq 0$ ).

The parameters of the utility functions in Eq. 14 and 15 correspond, in the given order, to the sequential outcomes of the following decision tree:

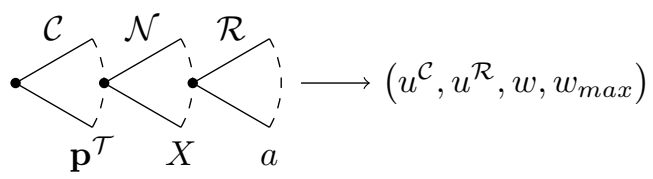

The tariff $\mathbf{p}^{\mathcal{T}}$ is determined by the customer $(\mathcal{C})$; the imbalance $X$, specified as the absence of energy, is determined by nature $(\mathcal{N})$; and the action $a$ is determined by the retailer $(\mathcal{R})$.

Furthermore, we define two variables for social welfare. The realised social welfare $w(a, X)$ does not depend on $\mathbf{p}^{\mathcal{T}}$-only on the action $a$ and imbalance $X$-and is given by:

$$
\begin{aligned}
w(a, X) & =u^{\mathcal{R}}+u^{\mathcal{C}} \\
& =\left(\mathbf{p}^{\mathcal{R}}-\mathbf{p}^{\mathcal{C}}\right) \cdot \mathbf{q}(X) \cdot[a=1]
\end{aligned}
$$

whereas the maximum obtainable social welfare $w_{\max }$ depends only on the imbalance $X$, and is given by:

$$
w_{\max }(X)=\left[\left(\mathbf{p}^{\mathcal{R}}-\mathbf{p}^{\mathcal{C}}\right) \cdot \mathbf{q}(X)>0\right]
$$

Any resource allocation that does not result in maximum social welfare shows an inefficiency. This is normal given a stochastic setting and given that the allocation mechanism cannot capture all private values. We compare the performance of our tariffs $\mathbf{p}_{\text {con }}^{\mathcal{T}}$ and $\mathbf{p}_{\text {inn }}^{\mathcal{T}}$ on the basis of their average allocation efficiency $\eta$, using:

$$
\eta=\frac{\bar{w}}{\bar{w}_{\max }}
$$

where $\bar{w}$ is the average social welfare over a number of sequential timeslots $j$, each with a stochastic imbalance $X_{j}$.

\footnotetext{
${ }^{7}$ The function $X(t)$ contains all relevant information concerning the imbalance. The function itself describes credit history, while its first three derivatives describe energy levels, power flows and ramp rates.
}

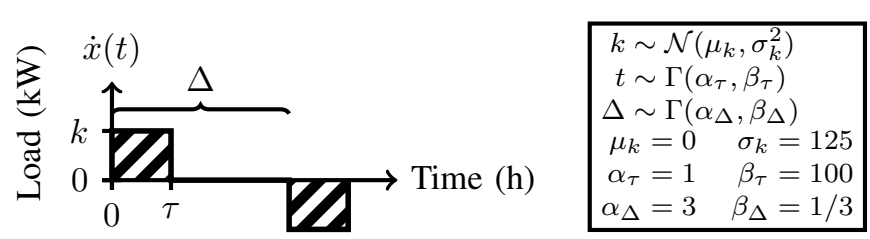

Fig. 4: Symmetric imbalance modelled as a load of size $k$ (in $\mathrm{kW}$ ) and duration $\tau$ (in $\mathrm{h}$ ), shifted by a duration $\Delta$ (in $\mathrm{h}$ ). The average load takes 0.1 hours and is shifted by 9 hours.

Settings: Stochastic simulations were repeated with identical seeds for the comparison between an effort-based tariff for loading (conventional tariff) and an effort-based tariff for both loading and stalling (innovative tariff). Each simulation comprised a repeated game, in which the customer uses a pattern search through the available tariff space-starting at its private costs - to maximise its expected utility. Each stage game comprised a 100 encountered imbalances drawn from a 3-parameter distribution as shown in Figure 4. Each imbalance is symmetric in the sense that a change in load is followed by an equal change in the opposite direction at a later point in time. This provides a simple yet very useful model of imbalances: any forecasted imbalance can be broken down into a number of symmetric imbalances, plus some residual (asymmetric) imbalance that is shifted so far to the future that it falls within an ahead market. Private value parameters are stated in Table II. The first row of $\mathbf{p}^{\mathcal{R}}$ corresponds to the retailer's expected costs for using its own portfolio of energy resources; the second row corresponds to the retailer's expected costs for using the imbalance market.

Results: Figure 5 shows the customer's search over the round-trip load and stall prices to find a good tariff. ${ }^{8}$ Shown searches start by offering DR services at cost price, i.e. at the customer's private costs. A customer using the conventional tariff does not charge according to storage effort $S_{x}$, and changes only the price of its load effort $S_{\dot{x}}$, achieving an average utility of $€ 13$ per imbalance. A customer using the innovative tariff that includes duration proportional pricing has a second degree of freedom to optimise its profit. Our initial search method yields an average utility of $€ 18$ per imbalance. As a result, the customer asks for a lower price

\begin{tabular}{|c|c|c|c|c|c|}
\hline & $S_{x}^{\uparrow}$ & $S_{x}^{\downarrow}$ & $S_{\dot{x}}^{\uparrow}$ & $S_{\dot{x}}^{\downarrow}$ & \\
\hline & $€ / \mathrm{MWh}^{2}$ & $€ / \mathrm{MWh}^{2}$ & $€ / M W h$ & $€ / M W h$ & \\
\hline $\mathbf{p}^{\mathcal{C}}=\langle$ & 5 & 5 & 60 & $60\rangle$ & (20) \\
\hline $\mathbf{p}^{\mathcal{R}}=$ & $\begin{array}{c}20 \\
0\end{array}$ & $\begin{array}{c}20 \\
0\end{array}$ & $\begin{array}{c}10 \\
150\end{array}$ & $\begin{array}{c}10 \\
150\end{array}$ & (21) \\
\hline
\end{tabular}
for load services and a higher price for stall services, which better reflects the structure of its private costs. Moreover, the

\footnotetext{
${ }^{8}$ The round-trip load price is the sum of prices for upload and download effort.
}

TABLE II: Private value parameters of the customer $\mathcal{C}$ and retailer $\mathcal{R}$. 


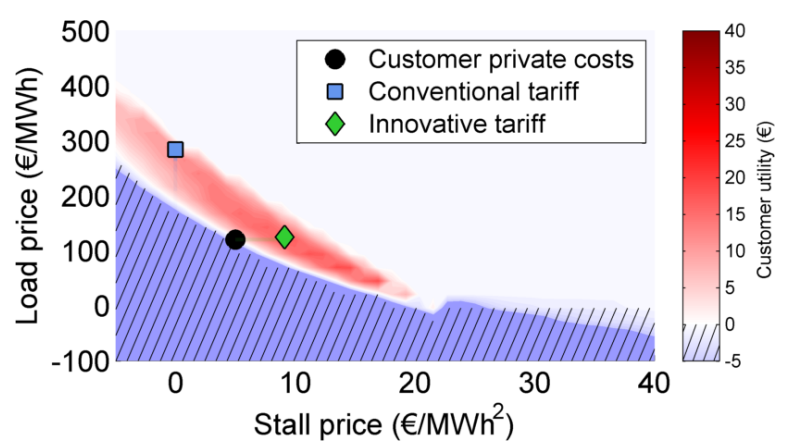

Fig. 5: Customer search over the available tariff family.

customer is able to learn a good pricing strategy for its services simply by interaction with its retailer.

Figure 6 shows the retailer's and customer's utility together with the socially best allocation of resources. The latter corresponds to the mechanism's Pareto front of economic surplus between the retailer and the customer. The customer's final innovative tariff yields a more efficient activation of its DR services than its final conventional tariff.

Finally, Figure 7 shows the effective round-trip MWh price as a function of storage time. Markers indicate the required storage time for a (random) selection of imbalances at the private costs for which they were resolved. With perfect knowledge, the customer may seek to set the innovative tariff at the retailer's private values. At that set of prices, the customer would be able to extract the most surplus out of the mechanism. Given imperfect knowledge about private values as well as expected imbalances, the customer's search converges to the prices as shown. This is a risk averse tariff, since it is always above the customer's private costs.

\section{CONClusion}

Balancing markets should level the playing field between technologies with various cost structures, and accommodate broader pricing structures. This paper contains three contributions to that end, which we elaborate in Section II. In Section III we present our service model for power balancing, encompassing e.g. ramping, production and storage. We argue that variable costs of storage and DR services scale both with

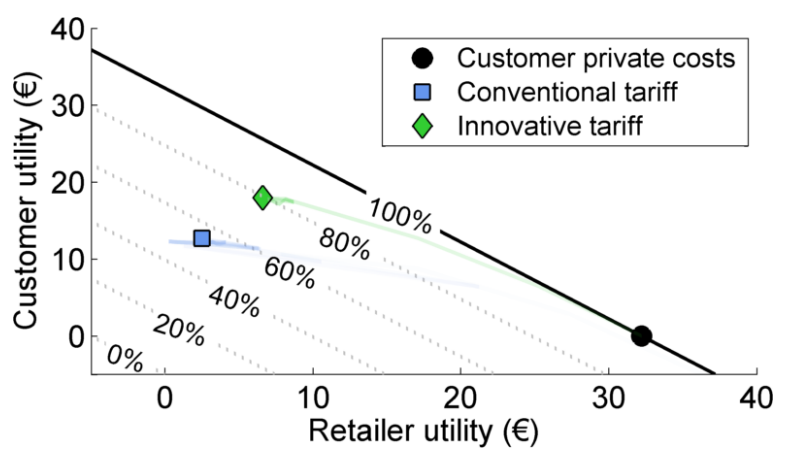

Fig. 6: Distribution of individual welfare per imbalance, and allocation efficiency of selected social indifference curves.

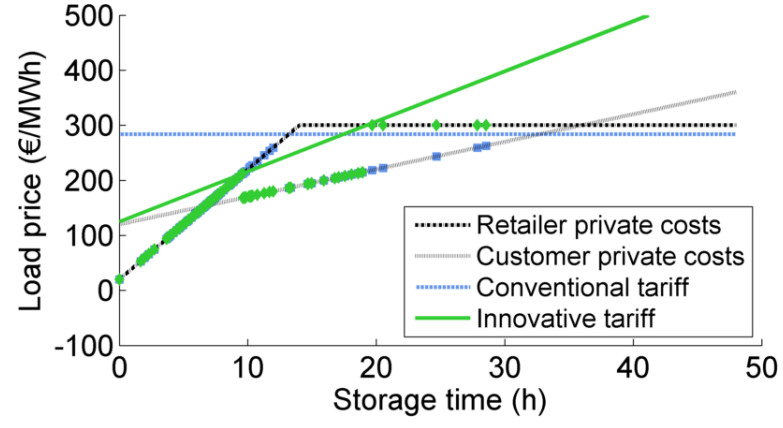

Fig. 7: Effective round-trip MWh price for storage durations up to 48 hours, including a selection of encountered imbalances.

the amount of stored or shifted electrical work (in $\mathrm{kWh}$ ), and with the duration of the shift (in h). That is, the effort of storage and DR is proportional to costs in $€ / \mathrm{kWh}$ per hour, i.e. in $€ / \mathrm{kWh}^{2}$. We also formally relate the relevant unit for these variable costs, stall effort (in $\mathrm{kWh}^{2}$ ), to the concept of absergy. In Section IV we investigate an alternative pricing structurefor a DR mechanism between a retailer and a customer - that explicitly includes the variable costs of stalling. This allows us to capture e.g. energy dissipation losses and opportunity costs for storage and DR. Both players benefit from our pricing structure, making the activation of DR services more efficient when compared to pricing in $\mathrm{kWh}$ alone.

Future work may extend our results to multiple customers while motivating truthful bidding, e.g. using a Vickrey type mechanism that may decrease the price of anarchy and further increase the activation efficiency. This may result in a new merit order of electricity services-focusing on storage instead of production-that ranks the storage capacity of flexible resources according to their marginal costs of stalling.

\section{REFERENCES}

[1] B. Edvardsson, A. Gustafsson, and I. Roos, "Service portraits in service research: a critical review," International Journal of Service Industry Management, vol. 16, no. 1, pp. 107-121, 2005.

[2] M. M. Roggenkamp and F. Boisseleau, Eds., "The regulation of power exchanges in europe," in Energy \& Law, vol. 2. Intersentia, 2005.

[3] G. Heffner, "Loads providing ancillary services: Review of international experience," Lawrence Berkeley National Laboratory, 2008.

[4] S. Mann, R. Janzen, M. A. Ali, P. Scourboutakos, and N. Guleria, "Integral kinematics (time-integrals of distance, energy, etc.) and integral kinesiology," pp. 270-272, 2014.

[5] D. Jeltsema, "Memory Elements: A Paradigm Shift in Lagrangian Modeling of Electrical Circuits," ArXiv e-prints, Jan. 2012.

[6] M. Zukerman, "Introduction to queueing theory and stochastic teletraffic models," arXiv preprint arXiv:1307.2968, 2013.

[7] Universal Smart Energy Framework Foundation, "USEF: The Framework Specifications 2015," nov 2015.

[8] R. De Coninck and L. Helsen, "Bottom-up quantification of the flexibility potential of buildings," in 13th Int. Conf. of the International Building Performance Simulation Association (IBPSA), 2013.

[9] S. Mathieu, "Flexibility services in the electrical system," Ph.D. dissertation, University of Liège, Belgium, 2016.

[10] B. Zakeri and S. Syri, "Electrical energy storage systems: A comparative life cycle cost analysis," Renewable and Sustainable Energy Reviews, vol. 42, pp. 569-596, 2015.

[11] C. Zhang, D. Yi, N. C. Nordentoft, P. Pinson, and J. Østergaard, "Flech: A danish market solution for dso congestion management through der flexibility services," Journal of Modern Power Systems and Clean Energy, vol. 2, no. 2, pp. 126-133, 2014. 